\title{
Cyclophosphamide-Induced Hyponatremia in a Patient With Diabetes Insipidus
}

\author{
Rachel A. Steinman ${ }^{a}$, Sara E. Schwab ${ }^{\text {, }}$ Kashif M. Munira, b
}

\begin{abstract}
Cyclophosphamide has been previously observed to induce hyponatremia. The mechanism remains unclear. Cyclophosphamide may produce a syndrome of inappropriate anti-diuretic hormone-like phenomenon through impairment of the kidney's ability to dilute urine. Whether cyclophosphamide or its metabolites have a direct effect on the kidney, a vasopressin-like effect on the kidney, or cause vasopressin release is unknown. A 29-year-old man with intracranial germinoma diagnosed at age 11 treated primarily with chemo-radiation developed a recurrence 17 years later and was noted to have panhypopituitarism with resultant central diabetes insipidus. He was admitted for chemotherapy with cisplatin, cyclophosphamide and mesna. His admission serum sodium was normal, but he became hyponatremic while undergoing chemotherapy with cyclophosphamide and mesna in conjunction with the initiation of intravenous hydration with $5 \%$ dextrose $/ 0.45 \%$ normal saline despite withholding two doses of desmopressin (DDAVP). The sodium eventually normalized after administration of $20 \mathrm{mg}$ intravenous furosemide. A similar episode occurred several weeks later, while again receiving cisplatin, cyclophosphamide and mesna chemotherapy. He again became hyponatremic despite receiving isotonic saline fluids and withholding DDAVP during cyclophosphamide treatment. Serum sodium did not improve with three doses of $10 \mathrm{mg}$ intravenous furosemide but improved instead with sodium chloride tablets. The mechanism of cyclophosphamide-induced hyponatremia remains unknown. Given that this patient's central diabetes insipidus prevents him from secreting increased amounts of anti-diuretic hormone, hyponatremia is likely induced by a direct nephrogenic effect of cyclophosphamide or its metabolites.
\end{abstract}

Keywords: Cyclophosphamide; Hyponatremia; Diabetes insipidus

Manuscript accepted for publication November 19, 2015

a Division of Endocrinology, Diabetes and Nutrition, University of Maryland Medical Center, Baltimore, MD, USA

${ }^{\mathrm{b}}$ Corresponding Author: Kashif M. Munir, Division of Endocrinology, Diabetes and Nutrition, University of Maryland School of Medicine, 827 Linden Avenue, Floor 2 South, Baltimore, MD 21201, USA.

Email: kmunir@medicine.umaryland.edu

doi: http://dx.doi.org/10.14740/jem319w

\section{Introduction}

It is well known that cyclophosphamide can result in hyponatremia. The mechanism, however, by which cyclophosphamide induces hyponatremia is yet to be fully elucidated. There is one prior case report that discusses the anti-diuretic effects of cyclophosphamide in an 8-year-old girl with established central diabetes insipidus [1]. Our case of a 29-year-old gentleman also with central diabetes insipidus and resultant cyclophosphamide-induced hyponatremia adds to the limited literature and suggests that cyclophosphamide or its metabolites must have a direct renal effect resulting in hyponatremia.

\section{Case Report}

A 29-year-old man with a history of intracranial germinoma diagnosed at age 11 was primarily treated with chemoradiation and subsequently developed a recurrence 17 years later. He developed panhypopituitarism with resulting central diabetes insipidus requiring desmopressin (DDAVP). He was admitted for chemotherapy with cisplatin, cyclophosphamide and mesna (Fig. 1). His admission serum sodium was 142 $\mathrm{mmol} / \mathrm{L}$ (normal range: 136 - 145). His serum sodium declined slowly over the course of a few days to $135 \mathrm{mmol} / \mathrm{L}$ while receiving cisplatin and his home dose of DDAVP. A significant decline in serum sodium to $125 \mathrm{mmol} / \mathrm{L}$ occurred while undergoing chemotherapy with cyclophosphamide and mesna in conjunction with the initiation of intravenous hydration with $5 \%$ dextrose $/ 0.45 \%$ normal saline despite withholding two doses of DDAVP. There was an accompanying decline in serum osmolality (osm) from 282 to $264 \mathrm{MoM} / \mathrm{kg}$ (normal range: 275 - 295). Urine sodium was $102 \mathrm{mmol} / \mathrm{L}$ and urine osm was $308 \mathrm{MoM} / \mathrm{kg}(50$ - 1,200) with a corresponding serum sodium of $126 \mathrm{mmol} / \mathrm{L}$, suggesting anti-aquaresis. With $20 \mathrm{mg}$ intravenous furosemide, serum sodium improved to $137 \mathrm{mmol} / \mathrm{L}$ with an associated urine sodium of $43 \mathrm{mmol} / \mathrm{L}$ and urine osm of $132 \mathrm{MoM} / \mathrm{kg}$. The serum sodium peaked at $142 \mathrm{mmol} / \mathrm{L}$. The hyponatremia occurred within $6 \mathrm{~h}$ after administration of cyclophosphamide and lasted approximately $35 \mathrm{~h}$.

A similar episode occurred several weeks later, while again receiving cisplatin, cyclophosphamide and mesna chemotherapy (Fig. 2). The patient's serum sodium declined from 


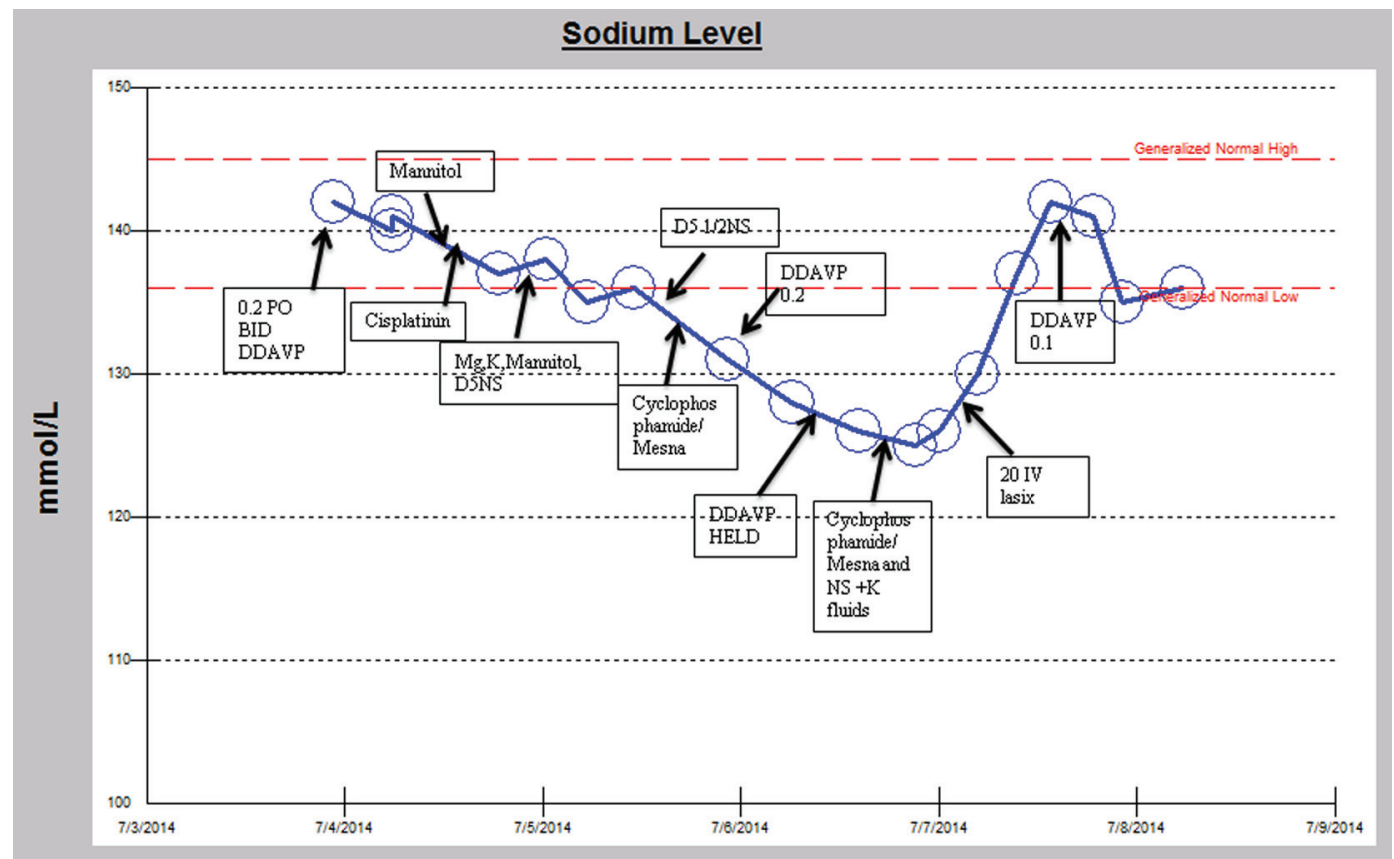

Figure 1. Sodium trend and relevant medication administration over the initial hospital course.

$141 \mathrm{mmol} / \mathrm{L}$ to a nadir of $122 \mathrm{mmol} / \mathrm{L}$ and serum osmolality declined from 288 to $258 \mathrm{MoM} / \mathrm{kg}$, despite receiving intravenous isotonic saline fluids and holding DDAVP after cyclophosphamide was initiated. The hyponatremia occurred within $6 \mathrm{~h}$ after receiving the cyclophosphamide. Urine osm was 654 $\mathrm{MoM} / \mathrm{kg}$ concurrent with a serum sodium of $130 \mathrm{mmol} / \mathrm{L}$ suggesting anti-aquaresis. Subsequently in the hospital course, when the sodium reached a nadir of $122 \mathrm{mmol} / \mathrm{L}$, the anti- diuretic hormone level was undetectable. Serum sodium did not improve with three doses of $10 \mathrm{mg}$ intravenous furosemide but improved instead with sodium chloride tablets $4 \mathrm{~g}$ by mouth every $4 \mathrm{~h}$ to $134 \mathrm{mmol} / \mathrm{L}$ on discharge. The urine osm improved to $126 \mathrm{MoM} / \mathrm{kg}$ with a corresponding serum sodium increase to $132 \mathrm{mmol} / \mathrm{L}$ later in the hospital course during the upward trajectory of serum sodium. Hyponatremia resolved after approximately $55 \mathrm{~h}$.

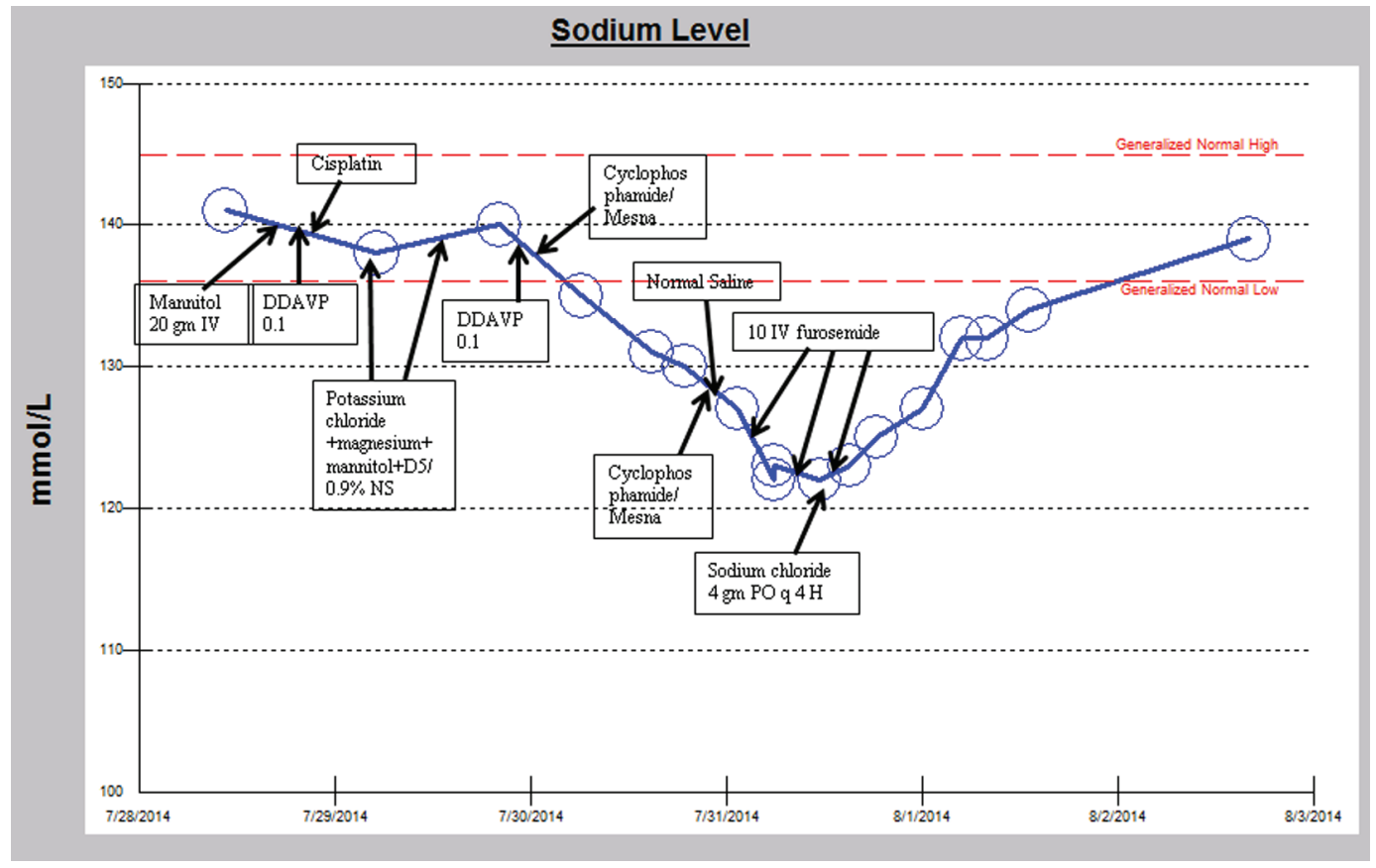

Figure 2. Sodium trend and relevant medication administration over the second hospital course. 


\section{Discussion}

Cyclophosphamide is an alkylating agent that has both antineoplastic and immunosuppressive properties. It is postulated to induce hyponatremia by limiting the renal excretion of water [2]. The exact mechanism by which cyclophosphamide produces this anti-diuretic effect is still under investigation. Some putative mechanisms are that cyclophosphamide or its metabolites induce vasopressin release, or directly act on the renal collecting tubules, or that cyclophosphamide metabolites have a vasopressin-like effect on the kidney [2-4]. A post-mortem exam in a patient with fatal, severe cyclophosphamideinduced hyponatremia showed loss of Herring's bodies and degranulation of hypothalamic neurosecretory organelles, suggestive of syndrome of inappropriate anti-diuretic hormone (SIADH) $[3,5]$. Some studies, including ours, however, have shown that plasma vasopressin levels are not elevated in patients who have received intravenous cyclophosphamide, thus suggesting more credibility to the theory that cyclophosphamide or its metabolites directly act on the kidney $[2,6]$. In addition to our patient, it has previously been reported that an 8 -year-old girl with central diabetes insipidus developed cyclophosphamide-induced antidiuresis [1], which suggests that cyclophosphamide does not cause vasopressin release since these patients would be unable to increase vasopressin levels. The undetectable anti-diuretic hormone level in our patient during an episode of hyponatremia also validates this point.

Recently, pre-clinical rodent studies have investigated the mechanism of this water retention and have suggested that cyclophosphamide may induce a drug-induced nephrogenic syndrome of inappropriate diuresis (NSIAD) by activating the vasopressin receptor 2 and upregulating aquaporin-2 channels and $\mathrm{Na}-\mathrm{K}-2 \mathrm{Cl}$ cotransporter type 2, resulting in decreased renal water excretion [7]. Our patient, who is unable to secrete vasopressin and yet experienced significant cyclophosphamide-induced hyponatremia, supports this mechanism of an NSIAD that does not require vasopressin to induce the electrolyte derangement.

Studies have shown that cyclophosphamide-induced hyponatremia frequently occurs 4 to $12 \mathrm{~h}$ after administration of intravenous cyclophosphamide and resolves in approximately $24 \mathrm{~h}$ [2]. Our patient experienced hyponatremia within $6 \mathrm{~h}$ after cyclophosphamide initiation but it resolved after a much longer period of time ( 35 and $55 \mathrm{~h}$ ). The prolonged duration of this hyponatremia is of unclear etiology. Perhaps, our patient was slower to metabolize this drug than other patients typically are or perhaps the furosemide, used with the aim of helping the hyponatremia, actually prolonged the hyponatremia. It is also possible that the DDAVP in our patient also prolonged the duration of hyponatremia. In the future, it may be beneficial to withhold DDAVP for a longer period of time prior to initiation of cyclophosphamide so as to take advantage of this patient's diabetes insipidus and invariable progression to polyuria and hypernatremia.

While it is well known that cyclophosphamide can result in hyponatremia, the mechanism by which this occurs is yet to be fully elucidated. Our case report, to our knowledge, presents only the second case in the literature of a patient with diabetes insipidus who developed cyclophosphamide-induced hyponatremia, thus providing a human example that lends further credence to pre-clinical data. In the future, cyclophosphamide or its metabolites may even be considered as prototypes for the development of new drugs to treat diabetes insipidus.

\section{References}

1. Campbell DM, Atkinson A, Gillis D, Sochett EB. Cyclophosphamide and water retention: mechanism revisited. J Pediatr Endocrinol Metab. 2000;13(6):673-675.

2. Lee YC, Park JS, Lee CH, Bae SC, Kim IS, Kang CM, Kim GH. Hyponatraemia induced by low-dose intravenous pulse cyclophosphamide. Nephrol Dial Transplant. 2010;25(5):1520-1524

3. Bruining DM, van Roon EN, de Graaf H, Hoogendoorn M. Cyclophosphamide-induced symptomatic hyponatraemia. Neth J Med. 2011;69(4):192-195.

4. Hwang SB, Lee HY, Kim HY, Lee ES, Bae JW. Lifethreatening acute hyponatremia with generalized seizure induced by low-dose cyclophosphamide in a patient with breast cancer. J Breast Cancer. 2011;14(4):345-348.

5. Harlow PJ, DeClerck YA, Shore NA, Ortega JA, Carranza A, Heuser E. A fatal case of inappropriate ADH secretion induced by cyclophosphamide therapy. Cancer. 1979;44(3):896-898.

6. Bode U, Seif SM, Levine AS. Studies on the antidiuretic effect of cyclophosphamide: vasopressin release and sodium excretion. Med Pediatr Oncol. 1980;8(3):295-303.

7. Kim S, Choi HJ, Jo CH, Park JS, Kwon TH, Kim GH. Cyclophosphamide-induced vasopressin-independent activation of aquaporin-2 in the rat kidney. Am J Physiol Renal Physiol. 2015;309(5):F474-483. 